INTERACTIVE CASE REPORT

\title{
A patient with suspected miscarriage is found to have hypertension, renal failure, and thrombocytopenia: case progression
}

\author{
Chris M Laing,, Rhys Roberts, ${ }^{1}$ Liz Lightstone, ${ }^{1}$ Alison Graham, ${ }^{3}$ Terry H Cook, ${ }^{4}$ Shaun Summers, ${ }^{1}$ Charles D Pusey ${ }^{5}$
}

\begin{tabular}{l}
\hline West London Renal and Transplant \\
Centre, Hammersmith Hospital \\
\hline 2Department of Medicine, \\
Hammersmith Hospital \\
${ }^{3}$ Department of Radiology, \\
Hammersmith Hospital \\
${ }^{4}$ Division of Investigative Science, \\
Imperial College London, \\
Hammersmith Hospital Campus, \\
London W12 ONN \\
5Division of Medicine, Imperial \\
College London, Hammersmith \\
Hospital Campus \\
\hline Correspondence to: C M Laing \\
christopher.laing@mac.com
\end{tabular}

BMJ 2007;335:44

doi: 10.1136/bmj.39239.478495.80

Contributors: CDP is guarantor. Accepted: 2 April 2007
This is the second of a three part case report where we invite readers to take part in considering the diagnosis and management of a real patient using rapid response on bmj. com. In three weeks' time we will report the outcome and summarise the responses.

We welcome contributions of interactive case reports. Cases should raise interesting clinical, investigative, diagnostic, and management issues but not be so rare that they appeal to only a minority of readers. Full details of criteria are available at: bmj.com/cgi/content/ full/326/7389/564/DC1
Last week (BMJ2007;334;1372, 30 June) we described the case of a 46 year old woman who presented after a possible miscarriage with severe hypertension, acute renal failure, alveolar infiltrates on chest radiography, thrombocytopenia, and grand mal convulsions.

Initially, she was given an intravenous infusion of lorazepam, frusemide, and a nitrate. A computed tomography scan of the brain excluded acute haemorrhage. She was then transferred to the renal unit of the Hammersmith Hospital.

On arrival, she remained severely hypertensive at 200/120 mm Hg. Fundoscopy demonstrated flame haemorrhages and papillo-oedema. She had severe peripheral and pulmonary oedema. Urine microscopy demonstrated red blood cells but no red cell casts. Her haemoglobin continued to fall to $8.4 \mathrm{~g} / \mathrm{l}$, with $11 \%$ reticulocytes, and her creatinine rose to $709 \mu \mathrm{mol} / \mathrm{l}$.

We thought that thrombocytopenia, red cell fragmentation on blood film, raised bilirubin concentrations, and raised lactate dehydrogenase concentrations were consistent with a diagnosis of microangiopathic haemolytic anaemia. The appearances on chest radiography were consistent with pulmonary oedema, although pulmonary haemorrhage could not be excluded.

Overall, the clinical features were consistent with accelerated hypertension, either "essential" or secondary to renal disease, renovascular disease, or endocrine disease. Scleroderma renal crisis usually presents with malignant hypertension. Systemic lupus erythematosis or vasculitis may cause not only renal failure but also seizures and pulmonary haemorrhage through direct organ involvement.

The microangiopathic haemolytic anaemia might have been a primary event-as in haemolytic uraemic syndrome or thrombotic thrombocytopenic purpura-with consequent renal failure and hypertension. Thrombotic thrombocytopenic purpura (and less often haemolytic uraemic syndrome) can cause neurological sequelae such as seizures.

We considered a pregnancy associated illness such as pre-eclampsia and eclampsia, which may cause many of these clinical features, particularly as part of the HELLP syndrome (haemolysis, elevated liver enzymes, and low platelets). These illnesses generally occur in the second half of pregnancy, but they have been reported earlier in pregnancy in association with extensive hydatiform changes or lupus anticoagulant.

Intravenous nitrates were continued. A tem- porary central venous haemodialysis catheter was inserted and she was haemodialysed for two hours with ultrafiltration of one litre of fluid. A pelvic ultrasound scan showed no retained products of conception. Magnetic resonance imaging of the brain was performed (figure). She was started on captopril at escalating doses. In addition to haemodialysis she had a seven day course of plasma exchange with one litre of frozen fresh plasma transfused during each exchange.

During this treatment further investigations were performed which clarified the underlying diagnosis.

\section{QUESTIONS}

1 What does posterior leucoencephalopathy found on the patient's magnetic resonance imaging scan indicate?

2 How quickly should you lower the patient's blood pressure?

3 What diagnostic tests would you do next?

Please respond through bmj.com, remembering that the patient is real and that she and her carers will read the response

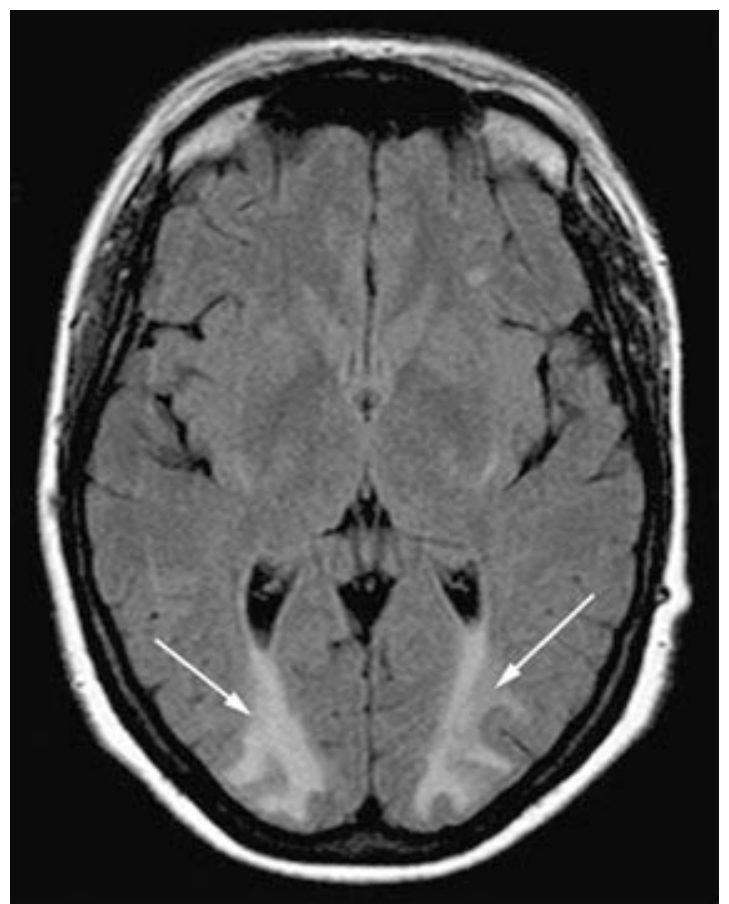

Magnetic resonance imaging scan of the patient's brain showing posterior leucoencephalopathy (arrows) 